hatte dank seiner internationalen Kontakte und Auslandserfahrungen mehr als 35 Jahre lang insgesamt über 830 überwiegend neuere Bibelausgaben in 507 Sprachen aus 125 Ländern gesammelt. Weil es bei den 233 deutschen Bibeln und vielen Ausgaben in anderen europäischen Sprachen starke Überschneidungen zum vorhandenen Bestand in Stuttgart gab, war für die WLB vor allem der außereuropäische Teil von Interesse. Am 19. August 2016 konnten 480 Bibeln mit dem Bücherauto in Königswinter abgeholt werden. Es handelt sich um Bibeln aus 91 Ländern in 428 Sprachen. Sowohl Vollbibeln als auch Teilausgaben sind vertreten. Einige Sprachen, vor allem im Bereich der Indianersprachen und afrikanischen Stammessprachen, sind bisher noch nicht in der Stuttgarter Bibel- sammlung vertreten. Vor allem für die Katalogisierung von Bibeln aus Asien werden Transkriptionen erforderlich sein. Alle neu aufgenommenen Bibeln werden durch den Provenienznachweis virtuell zusammengeführt. Die Sammlung Thesing lässt sich dadurch trotz der disparaten Aufstellung nach Sprachen jederzeit rekonstruieren.

Die Stuttgarter Bibelsammlung wird ihren Status als primäre Anlaufstelle für Anfragen und Studien zur Geschichte des Bibeldrucks durch diese Ergänzung weiter verbessern. Es ist davon auszugehen, dass einige der neu erworbenen Bibeln auch für Ausstellungen und die Fernleihe Verwendung finden.

\title{
Das Kriegstagebuch von Hans von Winterfeldt, 1914-1916
}

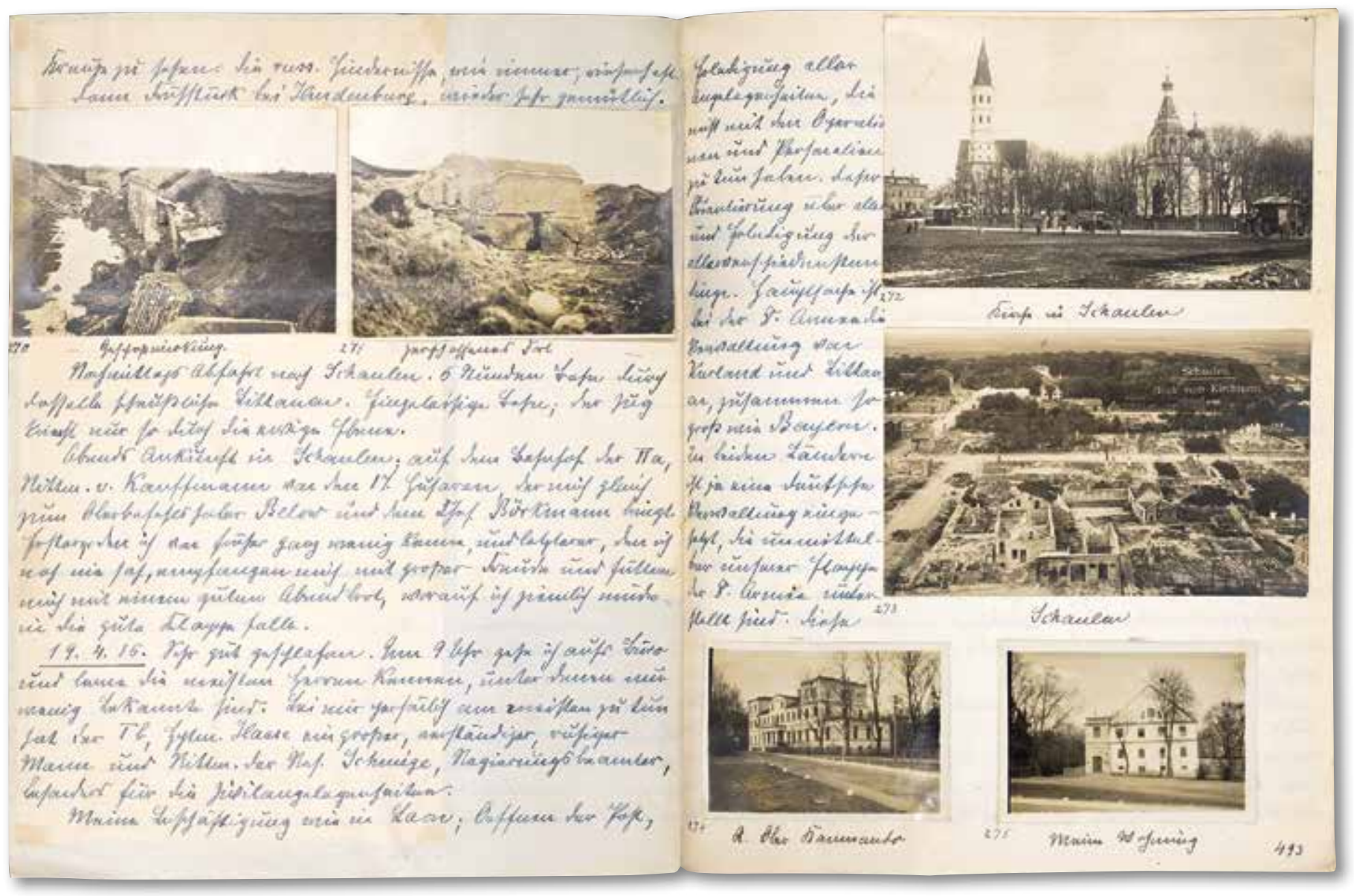


Am 27. Mai 2016 bot das Berliner Auktionshaus Jeschke van Vliet ein für die Bibliothek für Zeitgeschichte sehr interessantes Objekt an: das Kriegstagebuch von Hans von Winterfeldt, deutscher Generalleutnant und Oberquartiermeister im Ersten Weltkrieg. Bei dem Tagebuch handelt es sich um eine zentrale, bisher in der Fachwelt völlig unbekannte Quelle einer wichtigen Persönlichkeit des Ersten Weltkriegs. Es ist der Bibliothek gelungen, sich dieses bedeutende Ego-Dokument für ihre Lebensdokumentensammlung zu sichern. Über die Provenienz des Werkes ist leider nichts bekannt.

Winterfeldt hat seine „Erlebnisse "1 in chronologischer Reihenfolge in Sütterlinschrift notiert. Die vorliegende Fassung ist eine nach 1918 angefertigte Reinschrift seiner während des Krieges verfassten Tagebücher. ${ }^{2}$ Die Reinschrift auf Linienpapier umfasst insgesamt 637 paginierte Seiten. Im vorderen Einband findet sich ein Exlibris des Autors. Den Text ergänzen 371 eingeklebte und mit Unterschriften versehene Fotos von Kriegsschauplätzen, Kameraden, Paraden, Einsatzorten und Dienstwohnungen. Auch auf Karten wird verwiesen, die allerdings nicht vorliegen.

Hans Karl von Winterfeldt ( ${ }^{*}$ 29. März 1862 in Berlin, † 10. Oktober 1931 in Potsdam) war verheiratet mit Lilly von Bohlen-Halbach, der Schwester des Industriellen Gustav Krupp von Bohlen-Halbach. Winterfeldt stammte aus einer Adelsfamilie, die viele Militärs hervorgebracht hat. Zu seinen Vorfahren gehörte Hans Karl von Winterfeldt (1707-1757), preußischer Generalleutnant und enger Freund Friedrichs des Großen. Auch Hans von Winterfeldt schlug früh eine militärische Laufbahn ein. Er besuchte von 1891 bis 1894 die Kriegsakademie und diente in zahlreichen Regimentern und Korps, unter anderem im Generalstab des XIV. Armeekorps in Karlsruhe und im Infanterie-Regiment 114 in Konstanz. ${ }^{3}$

1) Winterfeldt, Hans von: Meine Erlebnisse im Weltkriege: 1914-1918, 1. Teil, [Potsdam] o.D., 637 S. (Württembergische Landesbibliothek, BfZ: N16.4).

2) Wentscher, Erich: Geschichte des Geschlechts von Winterfeld(t), Vierter Teil (Fünfter Band), Görlitz 1937, S. 253.

3) Ebd., S. $249 f$

4) Schwarte, Max (Hg.): Der große Krieg 1914-1918: in zehn Bänden, Leipzig 1921-1933.

5) Winterfeldt, Meine Erlebnisse, [Vorwort].

6) Eine Abschrift der Tagebucheinträge vom 8. bis 10. November 1918 befindet sich in einem Schreiben seines Bruders Detlof von Winterfeldt an den Staatssekretär der Reichskanzlei Hermann Pünder, 11. März 1932, Bundesarchiv Koblenz, Nachlass N 1005 Hermann Pünder, Nr. 174, BI. 63.

7) Sobbe, Friedrich von: Geschichte des Braunschweigischen Infanterie-Regiments Nr. 92 im Weltkriege 1914-1918, Berlin 1929, S. 17.
Winterfeldt war von 1916 bis 1918 Stabschef beim Generalgouverneur in Brüssel. Als solcher hatte er eine bedeutende Stellung innerhalb der deutschen Besatzungsverwaltung in Belgien inne. Die deutsche Verwaltung im besetzten Belgien schilderte Winterfeldt 1923 in einem umfangreichen Aufsatz, der im Überblickswerk „Der große Krieg 19141918 " ${ }^{4}$ erschien. Möglicherweise dienten Winterfeldts Tagebuchaufzeichnungen als Grundlage für diesen Beitrag, denn in seinem Vorwort schreibt er: „Vielleicht können sie einmal Material für die unparteiische Schilderung mancher Geschehnisse des Weltkrieges bilden, besonders für die Darstellung der Geschehnisse des General-Gouvernements in Belgien. " 5

Der vorliegende erste Teil seines Tagebuchs behandelt allerdings nicht seine Tätigkeit beim Generalgouvernement Belgien (1916-1918), mit der Winterfeldt meistens in Verbindung gebracht wird. Vielmehr berichtet er hier von seinen vorausgegangenen militärischen Verwendungen in den Jahren 1914-1916. Er beschreibt seine Einsätze bei verschiedenen Einheiten und seine Erlebnisse während zahlreicher Gefechte an der West- und Ostfront. Über den Verbleib des zweiten Bandes ist leider nichts bekannt. ${ }^{6}$ Die Bibliothek für Zeitgeschichte wäre sehr daran interessiert, auch diesen Band zu erwerben.

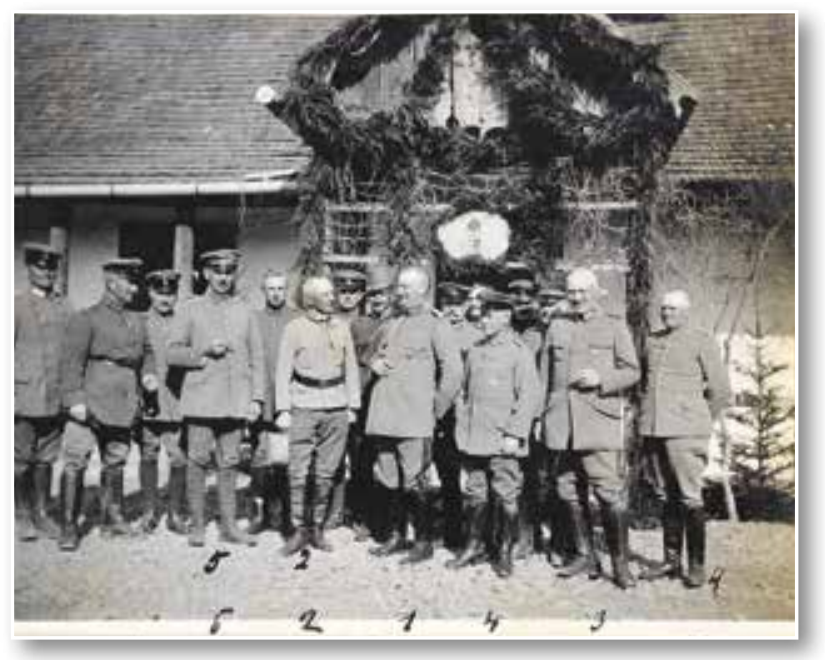

Abb. 2: Hans von Winterfeldt (Nr. 3) beim Frühstück des Divisionsstabs, 22. April 1915

Zu Beginn des Krieges wurde er von seinem Posten als Kommandeur des Braunschweigischen Infanterie-Regiments Nr. 92, den er seit 1912 innehatte, abberufen. ${ }^{7}$ Mit seiner neuen Einheit, 
der 37. Reserve-Infanterie-Brigade, erlebte er die Invasion Belgiens, die Schlacht an der Marne und den Übergang zum Stellungskrieg. Im Winter 1914/1915 diente er als Oberquartiermeister der 7. Armee, die zu dieser Zeit bei Charleville kämpfte. Im Frühjahr 1915 wurde er dann zur Südarmee an die Ostfront abkommandiert. Als Kommandeur der 8. Infanterie-Brigade war er an der großen Offensive der Mittelmächte beteiligt, welche die russischen Truppen aus Österreich-Ungarn vertrieb. Die Armeen Deutschlands und Österreich-Ungarns drangen im Sommer 1915 weit in russisches Territorium vor und besetzten Russisch-Polen, Litauen sowie Teile Lettlands und Weißrusslands. Nach einer Zwischenstation an der Westfront im Winter 1915/1916 kehrte er im Frühjahr 1916 an die Ostfront zurück. Als Oberquartiermeister der 8. Armee sollte er den Wiederaufbau Kurlands fördern und gleichzeitig für die wirtschaftliche Ausnutzung des Landes zu Gunsten der deutschen Kriegswirtschaft sorgen. ${ }^{8}$ Im Herbst 1916 folgte schließlich Winterfeldts Versetzung zum Generalgouvernement Belgien.

Im Tagebuch gibt es für fast jeden Tag einen eigenen Eintrag. Minutiös beschreibt Winterfeldt, was vorgefallen ist. Selbst seinen persönlichen Tagesablauf skizziert er zuweilen, so z.B. am 19. April 1916: „8 [Uhr] aufstehen und gutes Frühstück [...], Vormittags bis 12 [Uhr] arbeiten mit allen möglichen Leuten, die Anliegen haben; [...] 8 [Uhr abends] Essen, wieder im kleinen Kreise, danach Billardspiel bis 1/2 11 [Uhr], und dann Klappe"9. Als hoher Offizier führte Winterfeldt auch im Krieg ein komfortables Leben mit gutem Essen und Freizeit-Vergnügungen. Untergebracht war er in

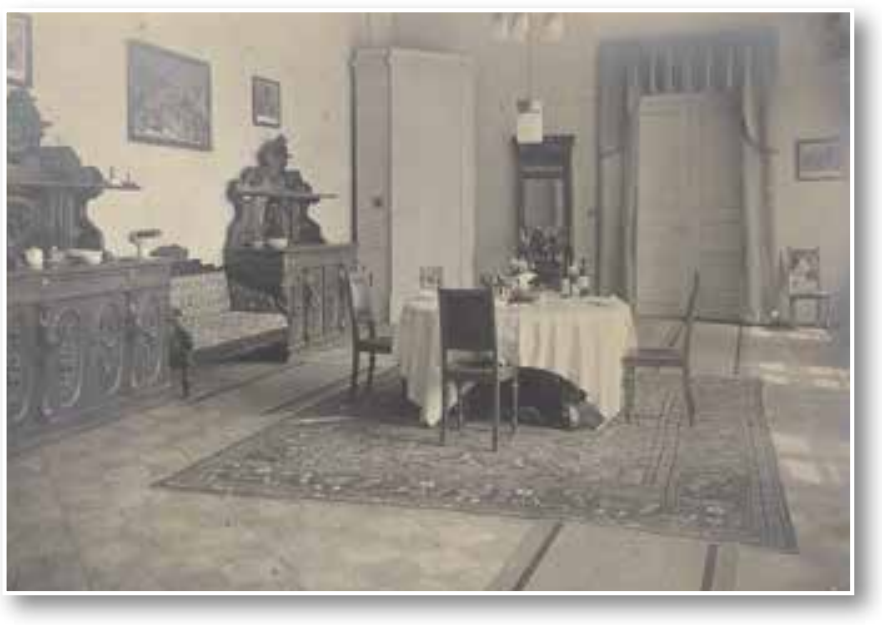

Abb.: 3: Von Winterfeldts Speisezimmer in Schloss Mitau, 1916 repräsentativen Unterkünften wie z.B. auf Schloss Mitau, der ehemaligen Residenz der Herzöge von Kurland. ${ }^{10}$

Interessant sind Winterfeldts Beobachtungen zur Kriegführung. An der Düna - im heutigen Lettland - konstatiert er am 10. Mai 1916, dass der Stellungskrieg an der Westfront mittlerweile wesentlich intensivere und schonungslosere Züge angenommen habe als an der Ostfront: „Die Russen müssen hier sehr friedlich sein. Schützengräben, Unterstände, alle Einrichtungen sind auf unserer Seite daher noch so, wie vor 11/2 Jahren in Frankreich und haben geradezu etwas rührendes. Von rückwärts geht man einfach über freies Feld in den Schützengraben; da würden einem die Franzosen mit Artillerie schön eins naufschießen. " 11

Die Folgen des schon fast zwei Jahre andauernden Krieges für den Umgang mit Menschenleben beschäftigen ihn. Als eine ehemalige Einheit von inm im Mai 1916 vor Verdun kämpft, freut ihn zwar, dass Höhe 304 erstürmt wurde. Er befürchtet jedoch gleichzeitig, dass solche Geländegewinne nur mit einem hohen Blutzoll zu erreichen sind, auch wenn dies die offiziellen Berichte nicht widerspiegeln würden: „Hoffentlich ist meinen guten Leuten nicht zu viel passiert; mäßige Verluste sollen sie nach dem Heeresberichte ja nur gehabt haben, aber in diesem Kriege sind die Begriffe sehr verändert und ,mäßig' ist schon immer eine ganze Masse."12

Auch über die Kriegsgegner macht er sich Gedanken: „Wir haben hier 4 russische Gefangene, welche die grobe Arbeit für unser Hauswesen machen, gute harmlose Kerle, die fleißig sind und unsern Burschen eine Menge Dreckarbeit abnehmen, Holz hauen u.s.w. Man versteht nicht, daß sie solche Viecher sein können wie sie in Ostpreussen waren." 13

An anderer Stelle wird Winterfeldts Haltung gegenüber der einheimischen Bevölkerung des besetzten Lettland deutlich. So schreibt er am 26. April 1916: „Lange Unterredung mit dem Kreis-

8) Wentscher, Geschichte, S. 252.

9) Winterfeldt, Meine Erlebnisse, S. 495.

10) Ebd., S. 525.

11) Ebd., S. 516

11) Ebd., S. 516

12) Ebd., S. 515

13) Ebd., S. 219 
hauptmann von Libau, Berkerer. [...] Der schimpft sehr auf die Civilverwaltung von Ober Ost in Schulangelegenheiten, weil diese besondre Schulen für die Letten eingerichtet hat, während es doch von größtem Nutzen wäre, [...] möglichst zu germanisieren; ich muß inm recht geben." 14

Am 1. Oktober 1916 erfährt Winterfeldt per Telegramm von seiner Berufung zum Chef des Stabes des Generalgouvernements Belgien. Er selbst ist überrascht von der Nachricht - „aber nicht unangenehm" - , eine wirkliche Erklärung für seine Abberufung hat er nicht: „Da muss irgendetwas plötzlich passiert sein. ${ }^{\prime 15}$

Zusammenfassend lässt sich festhalten, dass das vorliegende Kriegstagebuch von Hans von Winterfeldt auf einmalige Weise Einblick gibt in die Erfahrungen, die er während der Jahre 1914 bis 1916 sammelte. Aufgabe der Forschung ist es, diesen Erfahrungshorizont näher zu ergründen und her- auszufinden, welche Rückwirkungen diese Prägungen auf seine anschließende Tätigkeit in der deutschen Besatzungsverwaltung in Belgien hatten. Auch mit Blick auf die deutsche Besatzungspolitik in Kurland stellen Winterfeldts Notizen eine sehr wichtige Quelle dar, da die entsprechenden Aktenbestände des preußischen Kriegsministeriums im April 1945 bei einem Luftangriff zerstört wurden und nur wenige vergleichbare private Aufzeichnungen vorliegen. Schließlich vermitteln Winterfeldts Äußerungen auch einen Einblick in das Leben und die Perspektive eines hohen Militärs, der an unterschiedlichsten militärischen Auseinandersetzungen des Ersten Weltkriegs beteiligt war.

Das Kriegstagebuch Hans von Winterfeldts, 1. Teil, wurde in die Lebensdokumentensammlung der BfZ übernommen (Signatur N16.4). Es ist geplant, das Tagebuch zu digitalisieren und online zur Verfügung zu stellen.

Christian Westerhoff

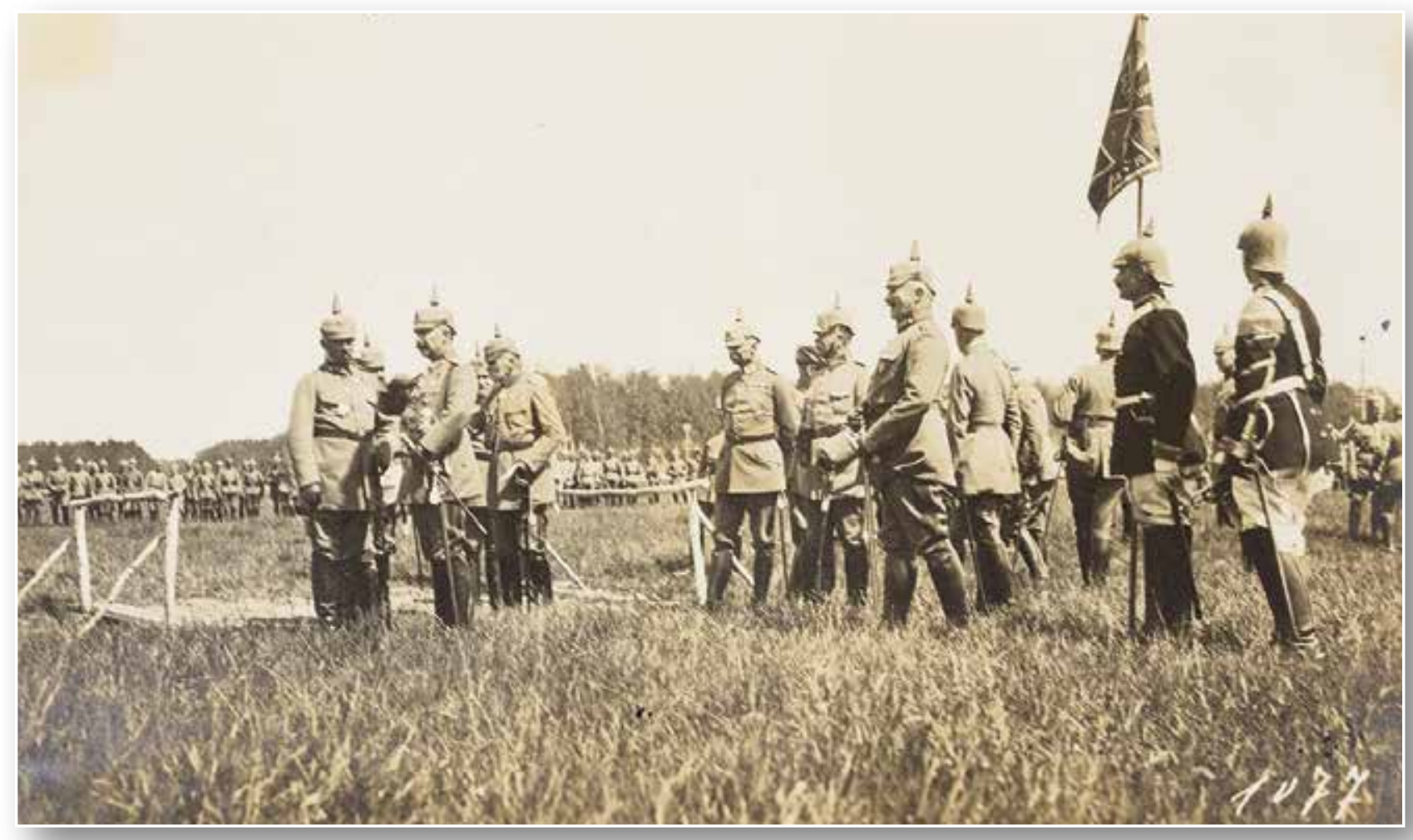

Abb. 4: Hans von Winterfeldt (rechts in dunkler Uniform) beim Besuch von Kaiser Wilhelm II. in Mitau am 30. Mai 1916 